\title{
In Situ Transmission Electron Microscopy of the Electrochemical Intercalation of Graphite in Concentrated Sulfuric Acid
}

\author{
B. C. Regan, E. R. White, Jared Lodico, Gavin Carlson, Nicolas Macro, and William A. Hubbard \\ Department of Physics \& Astronomy and California NanoSystems Institute, University of California, \\ Los Angeles, California 90095 USA
}

Graphite intercalation compounds, where some atom or molecule is inserted between the carbon layers of the host graphite, are attractive materials for charge storage. In commercial, rechargeable lithium ion batteries, for instance, the graphite anode intercalates and de-intercalates lithium as the battery is cycled [1]. In this work we present our in situ scanning transmission electron microscopy (STEM) observations of a model electrochemical intercalation system, namely graphite and concentrated sulfuric acid (98\% mass fraction $\mathrm{H}_{2} \mathrm{SO}_{4}$ ). We cycle the potential between two graphite/gold electrodes that are immersed in the sulfuric acid and sealed in a fluid cell designed for in situ TEM. The construction of the fluid cell and the graphite transfer process have been described previously [2].

Figure 1 shows evidence for the reversible intercalation of sulfate ions into graphite, and also for the direct imaging of the changing concentration of sulfate ions in the acid solution. Two consecutive raw STEM images from a ten-minute video acquisition are shown in panels A and B. As the scan raster, which scans lines left to right and the frame top to bottom, moves past the midway point of frame B, the voltage on the graphite sheet is taken from $+2 \mathrm{~V}$ relative to the graphite/gold counter electrode (outside the field of view) to $-2 \mathrm{~V}$. The timing and magnitude of this voltage change is recorded with sub-millisecond time resolution by digitizing the applied voltage (buffered) simultaneously with the output of the annular dark field (ADF) STEM detector (data not shown).

The STEM intensity changes are subtle and difficult to perceive in static, consecutive, unprocessed images, but they are easily visualized by constructing difference images as shown in the third panel $\mathrm{C}$. Even without the voltage data the timing of the polarity switch is clearly evident in the difference signal, which transitions from almost pure noise to a series of distinct, dark islands or pockets. The darkness indicates a decrease in scattering and thus a loss of material in these dark field images. Generally these pockets, which become correspondingly bright a half-cycle later, appear and persist for some number of cycles but are not uniformly active throughout the entire ten minute data acquisition.

While difference images give a detailed spatial map of the graphite regions most changed by the voltage cycling, complementary information can be obtained by averaging the raw signals over extended areas, or regions-of-interest (ROIs). Panel D of Figure 1 indicates three ROIs: ROI 0, 1 and 2, which are respectively away from, adjacent to, and directly on the graphite sheet. Panel E shows the intensity from these ROIs as a function of time during the period surrounding the acquisition times of frames A and B, which are indicated with arrows on the plot. ROI 0 , the negative control, shows no significant variation over the $100 \mathrm{~s}$ period plotted, while ROI 2 shows substantial variation as expected based on the position of the largest dark pocket in the difference image $\mathrm{C}$. Note, however, that even though the voltage variation was constant throughout the entire period plotted, the intensity variations in ROI 2 are markedly smaller or absent during the first 25 s. Evidently the repeated voltage cycling caused the 
chemical or mechanical activation of the graphite in ROI 2. ROI 1 shows perhaps the most interesting signal, namely significant peaks associated with the falling edges of the ROI 2 signal.

We interpret this data as follows. Away from the graphite sheet the column density of the sulfate ions is constant, but in certain regions the graphite intercalation causes the column density to change in synchronicity with the applied voltage. (Simultaneously acquired electrical transport data shows currents too large to be explained by the formation of an electrical double layer.) Adjacent to the graphite, de-intercalation produces a signal that looks like the derivative of the intercalation signal: as sulfate ions leave the sheet they produce a brief intensity pulse at the boundary before diffusing away. Thus changing concentrations of even relatively low- $Z$ ions - oxygen has $Z=8$ and sulfur has $Z=16$-can be directly mapped with ADF STEM [3].

\section{References:}

[1] M. Nie et al, Journal of Physical Chemistry C 117 (2013) p. 1257.

[2] B. C. Regan et al, Microscopy and Microanalysis 19 Suppl. 2 (2013), p. 454.

[3] E. R. White et al, ACS Nano 6 (2012), p. 6308.

[4] This work was supported by NSF DMR-1206849; STARnet, a Semiconductor Research Corporation program sponsored by MARCO and DARPA; and the Electron Imaging Center for NanoMachines at UCLA.

Figure 1. Dark field STEM images immediately before (A) and during (B) a reversal of the $2 \mathrm{~V}$ potential applied to the thin graphite sheet pictured. Frame (C) shows the difference image, with a yellow horizontal arrow indicating the point in the scan when the potential reversed. Frame (D) contains three yellow rectangles designating regions-of-interest (ROIs). The STEM intensity averaged over each ROI (arbitrary units) is plotted in frame (E) as a function of time (seconds).

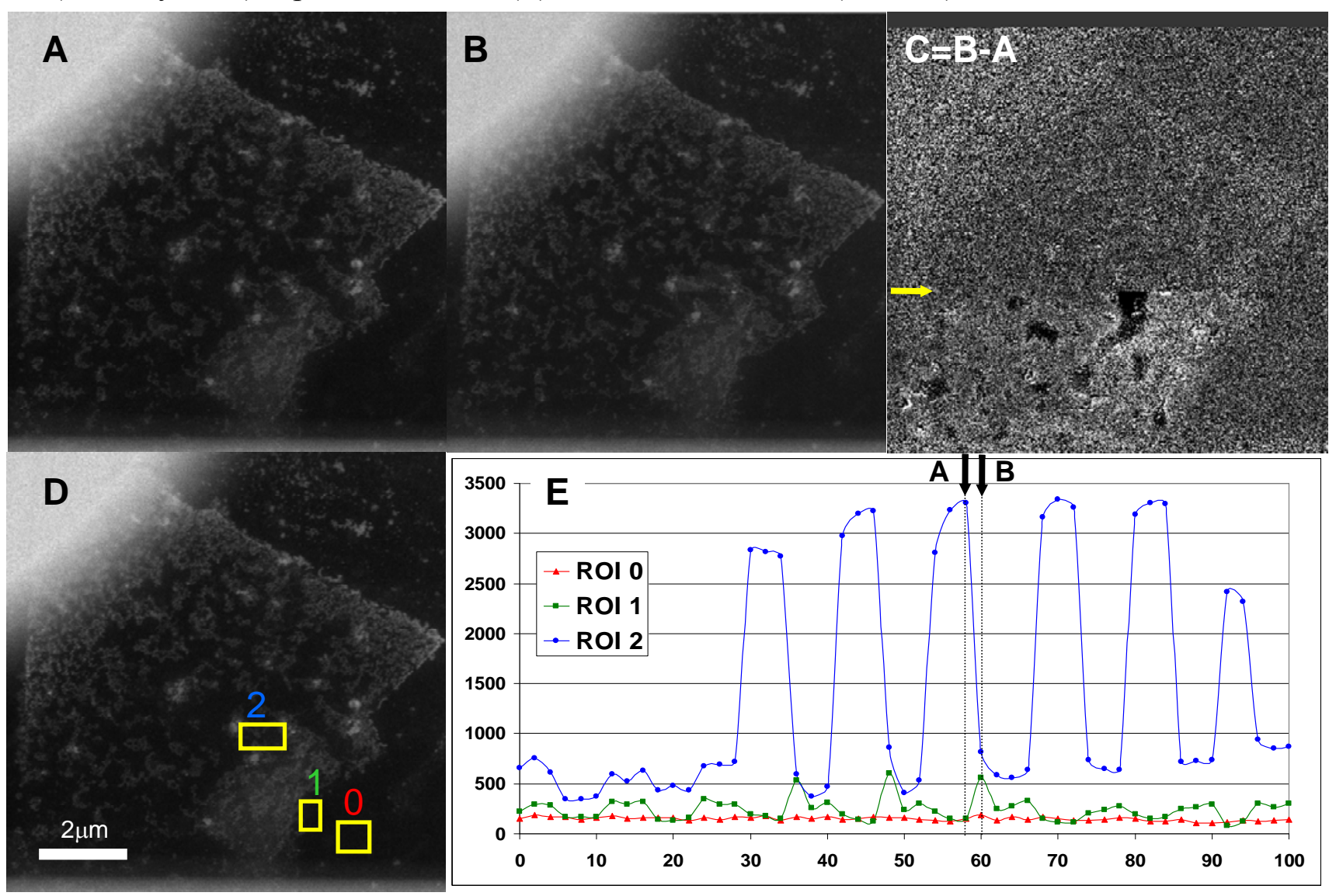

